\title{
Optimized Strategies for Smart Nuclear Search
}

\author{
Konstantin N. Borozdin, Alexei V. Klimenko, William C. Priedhorsky, Nicolas Hengartner, Charles C. Alexander, R. \\ Andres Cortez, Herbert G. Tanner, Xanthi Papageorgiou
}

\begin{abstract}
In many applications the time available for nuclear materials detection is short, the signals are weak, and the backgrounds are large, variable, and difficult to control. We develop optimal strategies for nuclear search in these difficult conditions, and demonstrate these strategies in experiments. Model-driven algorithms promise to reduce search time by order of magnitude, with increased reliability of detection and reduced number of false positives. These strategies can be applied to various nuclear search scenarios, for both mobile and stationary detectors, hand-held detectors and sensors on robotic platforms. Our methods can be used against nuclear smugglers and terrorists, for safeguards and non-proliferation treaty monitoring, as well as in other situations where radioactive sources need to be found.
\end{abstract}

\section{INTRODUCTION}

SPECIAL nuclear materials are weak emitters of radiation and Stherefore difficult to detect, especially in the presence of natural and artificial backgrounds. Success of detection depends largely on the proximity of the sensor and the time for measurement. A small detector up close outperforms a much larger detector at a distance (Fig.1, 2). When noise is dominated by statistical variations in background, a $1 \mathrm{~cm}^{2}$ detector at $1 \mathrm{~m}$ can obtain the same signal-to-noise ratio as a 1 $\mathrm{m}^{2}$ detector at $10 \mathrm{~m}$ range [1]. Mobile sensors can close the distance gap, enabling high sensitivity with low false alarm rates.

We need an optimized strategy for a moving detector that minimizes search time. Our objective is more efficient strategies for search when signal-to-noise is limited by the quantum noise of nuclear detection. Small number statistics and large backgrounds can make nuclear detection difficult. However if the backgrounds are known and the statistical behavior of their fluctuations are predictable, we can theoretically quantify the optimal approach to the search problems.

In classical statistical methods, the data are first collected and then the analysis is performed. For source detection at a single location, this means that we collect data for the entire allocated search time $T_{s}$ before making a decision about the presence or absence of a source, depending on whether or not a given threshold is crossed. However, a decision can typically be made at an earlier time $T_{d} \leq T_{s}$, if we can monitor the counts continuously. The basic idea is to consider two alternative hypotheses and, based on the strength of the

Manuscript received November 27, 2006. This work was supported in part by the Technical Development funds of the Los Alamos National Laboratory.

K. N. Borozdin (telephone: 505-665-1906, e-mail: kbor@lanl.gov), A. V. Klimenko, W. C. Priedhorsky, N. Hengartner, and C. C. Alexander are with the Los Alamos National Laboratory, Los Alamos, NM 87545 USA.

R. A. Cortez, H. G. Tanner, and X.Papageorgiou are with the Mechanical Engineering Department, University of New Mexico, Albuquerque, NM USA. measured signal, decide sequentially at times $t_{1}, t_{2}, \ldots$ between three options: 1) hypothesis Ho holds, 2) hypothesis $\mathrm{H}_{1}$ holds, or 3 ) more data are needed to decide between the two hypotheses. The principle of continuous real-time monitoring and making decisions constitutes the foundation of the classical sequential testing theory [2].

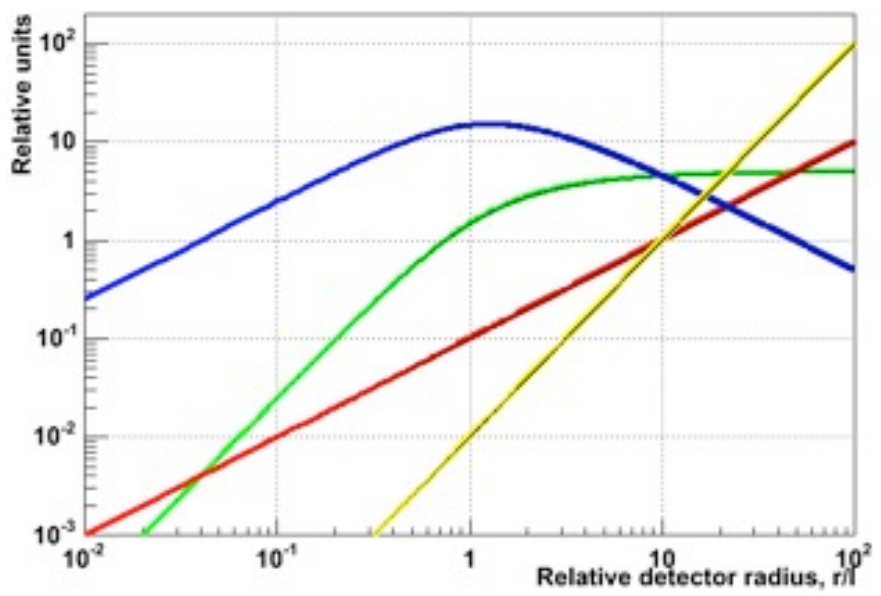

Fig. 1. Bigger detector does not guarantee more efficient detection for a point source. Source signal grows as solid angle (green line), while background grows proportionally to the detector area (red line), as a result, signal to noise ratio (blue line) has a maximum at a relative detector size (ratio of the detector radius to the detector-source distance) close to 1 . Meanwhile, cost of the detector usually grows proportional to the area (yellow line).

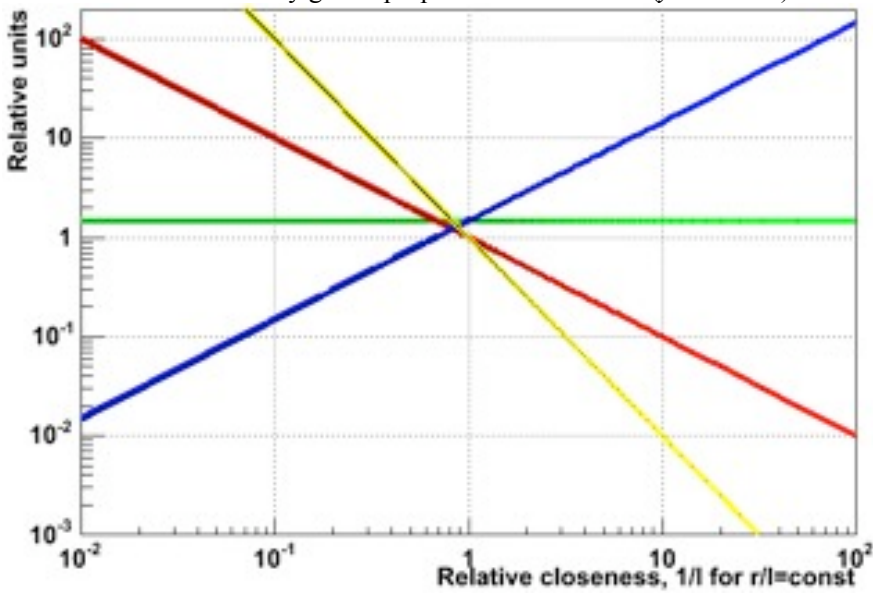

Fig. 2. Smaller detector close to the source outperforms the bigger one at the distance. For the same collected signal (green line), both background (red line) and cost (yellow line) decrease dramatically as the source gets closer, and signal to noise ratio (blue line) raises dramatically as a result.

\section{NuClEAR SEARCH StRATEGIES}

We showed in our previous work [3] that smart strategies are almost an order of magnitude more effective than naïve, uniform search. The simplest way to search for a radiation source is to scan the area uniformly, exposing each region for a fixed time interval. The standard deviation of the Poisson 
distribution is $\sigma=\sqrt{\mu \cdot t}$. By exposing it long enough, $\sigma$ becomes small relative to $\mu$, and one can separate the count rate distributions from the source and background. For an average expected background $\mu_{\mathrm{b}}$, signal $\mu_{\mathrm{s}}$ and exposure time $t$, the threshold of the number of observed counts can be set that satisfies the required false positive and false negative probabilities of the search outcome. of experiments. In the uniform search, the resources (sensors and exposure time) are allocated independently of the observation. When a few sources are present in a large area, most of the time is spent on measuring the background.

Application of the classical sequential testing theory allows one to save time by rejecting certain sequences of observation at early stages. The theory suggests stopping rules that allow for rejection of certain sequences of observations at early stages. Either positive or negative identification can be made based on the likelihood ratio $\mathrm{K}=\frac{P\left(N_{k} \mid S\right)}{P\left(N_{k} \mid B\right)}$, where $P\left(N_{k} \mid S\right)=\frac{\left(t_{k} \cdot \mu_{S}\right)^{N_{k}}}{N_{k} !} \cdot e^{-t_{k} \cdot \mu_{S}}$ is the probability to observe $\mathrm{N}_{\mathrm{k}}$ counts within time period $\mathrm{t}_{\mathrm{k}}$ from a source with an average count rate $\mu_{\mathrm{k}}$.

The stopping rules are determined from the desired false negative and false alarm rates. Real-time updating of a physical model of the radiation field is essential to this strategy, allowing optimal resource management and sensor cooperation.

One can do even better in the situation of multiple fast scans, followed by dwells on tentative detections. In this case we can search the area by allocating search time at each location as suggested by the prior probability of source detection. The prior initially can be taken either uniform or biased, incorporating any prior knowledge about the area. The

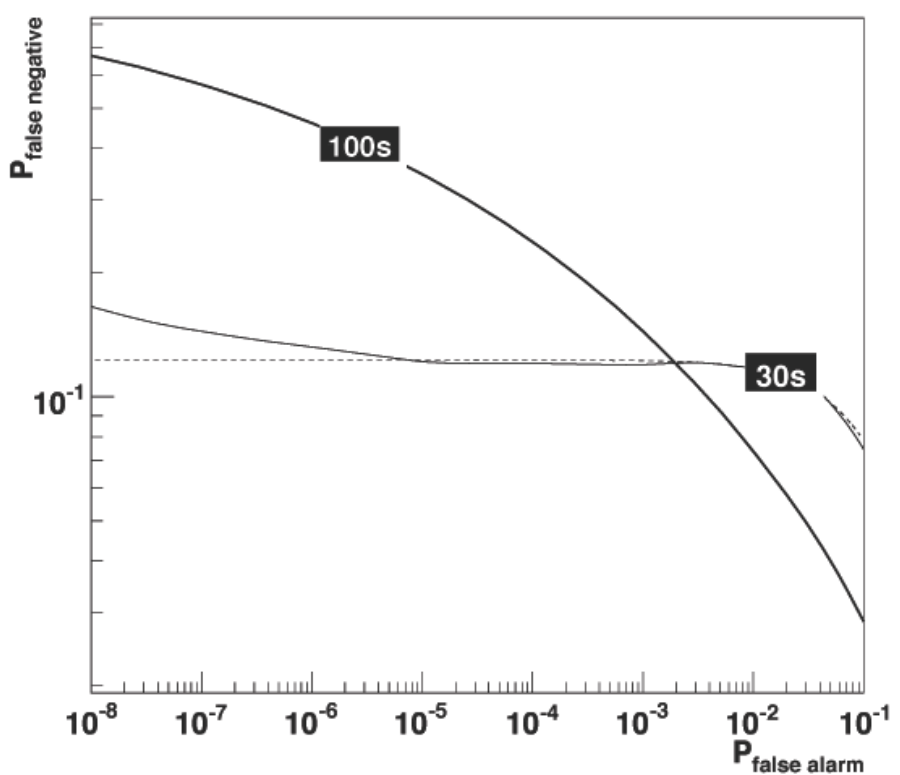

Fig. 3: Smart nuclear detection techniques yield better performance at reduced inspection time. Uniform inspection strategy (thick solid line) is compared with sequential strategy (thin solid line) and multiple-scan Bayesian strategy (dashed line). probability of that the set of observations $N=\left\{\mathrm{N}_{0}, \mathrm{~N}_{1}, \ldots \mathrm{N}_{\mathrm{k}}\right.$, ... was obtained as a result of source being present in location $\mathrm{k}$ is: $P(N \mid H=k)=P\left(N_{k} \mid S\right) \cdot \prod_{j \neq k} P\left(N_{j} \mid B\right)$, whereas the probability that there is no source present in the area is: $P(N \mid H=\varnothing)=\prod_{j} P\left(N_{j} \mid B\right)$.

At the next sweep we would like to spend more time at the locations, where $\frac{P(N \mid H=k)}{P(N \mid H=\varnothing)}=\frac{P\left(N_{k} \mid S\right)}{P\left(N_{k} \mid B\right)}=\max$, therefore for i-th sweep we allocate time as $t_{i}(k) \propto P_{i-1}(N \mid H=k)^{\alpha}$.

Strategies outlined here provide much better performance in shorter time, especially reducing number of false positives, as necessary for many nuclear search applications (Fig.3).

\section{ROBOTIC HARDWARE IMPLEMENTATION}

We demonstrate the feasibility of our strategies in a series of experiments, using mobile detectors. Experimental implementation of the sequential search algorithm was performed using the Khepera II robot with a custom- built turret to interface the CsI radiation sensor (Fig. 4). The Khepera II robot features a Motorola $68331,25 \mathrm{MHz}$ processor with 512 Kbytes RAM and 512 Kbytes Flash memory, running our embedded $\mathrm{C}$ code that implements the sequential search motion controller. We use three analog inputs provided by the I/O turret, and communicate with it through RS232. Data acquisition software is also developed, allowing for realtime 1-D and 2-D radiation mapping during experimentation.

The Khepera II is equipped with a CsI radiation sensor. Gamma-rays that pass through the CsI crystal have a probability of depositing some or all of their energy. The deposited energy goes into excitement of the electrons into higher energy levels that decay with characteristic lifetime and emit visible light. The $4 \mathrm{~cm}$ long and $1.2 \mathrm{~cm}$ in diameter cylindrical CsI crystal was encapsulated into the Al casing with the Hamamatsu S3509 pin photodiode mounted on it to detect light induced in the crystal by passing photons. The sensor was assembled by the Alpha Spectra, Inc. The pulse generated by the diode is very weak and needs to be amplified. We used the A250 preamplifier with external FET from Amptek. The pulse is then shaped through a four-stage shaping amplifier based on operational amplifiers. On the output of the amplifier we get almost Gaussian pulse, which peak corresponds to the energy that was deposited by the gamma-ray in the active region of the detector. The pulse is processed using digital board that is based on low power, high speed, 8-bit National Semiconductor ADC08200 and Altera Cyclone 2910 FPGA. The FPGA can be programmed to perform all necessary pulse post-processing. We have developed FPGA code to perform peak finding and pulse counting. We estimated the total power consumption of the electronics to be below $200 \mathrm{mAh}$ at $6 \mathrm{~V}$, allowing us to power them for several hours with four rechargeable digital camera batteries. The pin photodiode is in reverse bias and consumes negligible amount of power (nAh at $25 \mathrm{~V}$ ). 


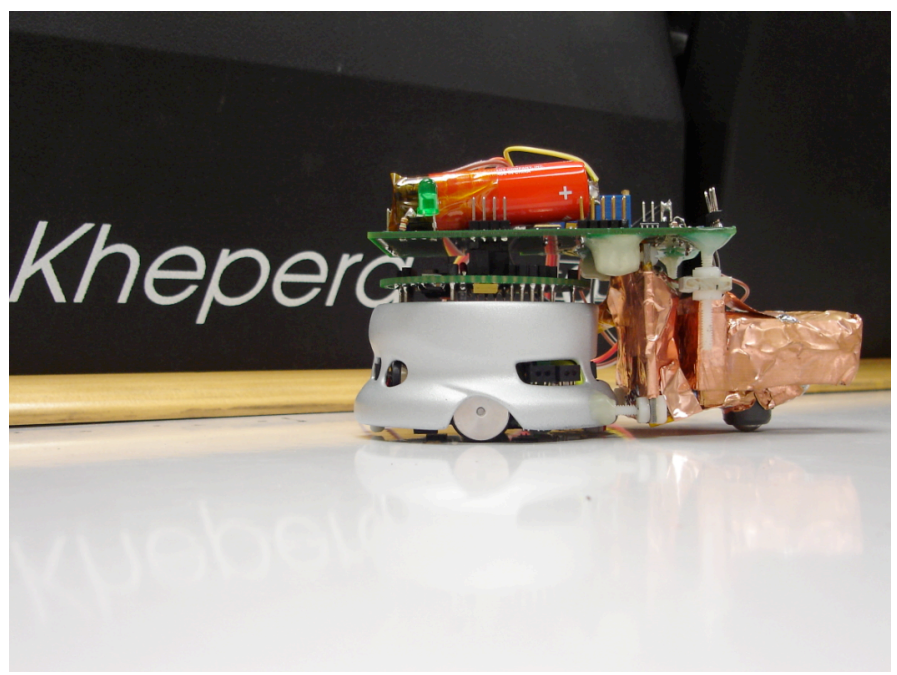

Fig. 4: A miniature robot Khepera from K-Team Inc., equipped with our gamma-sensor and data acquisition electronics. We use this robot/sensor combination in our demonstration experiments.

\section{EXPERIMENTAL SEQUENTIAL STRATEGY}

In a sequential search for a weak radiation source the space is divided into pixels and the sensor collects measurements at each for different time periods. These time periods are determined by the need to reach a statistically definitive conclusion on whether a radioactive source is present in the pixel. Once the decision is made, the sensor jumps to the next pixel. For real world applications we need to modify the method to make it applicable in a continuous space-time framework, where sensor cannot jump from one pixel to another and measurements are collected continuously. We approach this issue by regulating the velocity and acceleration of the moving sensor to approximate the discrete algorithm as close as possible. To speed up the search, our motion controller maintains a maximum scanning speed while the observed count rate is consistent with our model of natural background radiation. When the increase in the count rate is observed, the sensor decelerates to a level where the exposure time is sufficient to produce a definitive answer at a very high confidence level as to whether source is present there. As soon as the robot can reach a decision regarding the presence or absence of a source, it starts moving again at a maximal speed.

While robot is moving with the constant speed each location is exposed for the same time. During each sampling period $\Delta \mathrm{T}$, the counts recorded by the radiation detector are added up. At the end of each step, the sum is stored and the detector's buffer is cleared to begin recording the new sum. The rate of change (increase) of counts in the i-th step is estimated at the end of each time step as $\dot{C}=\frac{d c}{d t} \approx \frac{C_{i}-C_{i-1}}{\Delta T}$. When $\dot{C}$ is above a set threshold, the robot decelerates and continue to collect counts from the location of suspected source until its presence can be confirmed with high fidelity or refuted.

We have been able to detect radiation sources in $2 \mathrm{D}$ demonstration experiments using our movable sensor. Further steps include extensions to three-dimensional searches, the use of prior knowledge about the search area, as well as the introduction of Bayesian statistics for the on-line update of the world model. The latter will enable us to map arbitrary radiation levels over an area, rather than just confirm the presence of a source. We also plan to address a problem of navigation in the presence of some realistic obstacles.

\section{CONCLUSION}

Our strategies can be applied to various nuclear search scenarios, for both mobile and stationary detectors, hand-held detectors and sensors on robotic platforms. Our methods can be used against nuclear smugglers and terrorists, for safeguards and non-proliferation treaty monitoring, as well as in other situations where radioactive sources need to be found. Efficient nuclear search requires using advantages of close range detection. In the future nuclear search can be performed with a swarm of radiation sensors moving around on robotic platforms. Existing capabilities of robotic platforms are not adequate for the task, but they are rapidly improving. Dynamic interaction between data collection and knowledge extraction allows reduction in search time. Smart algorithms are needed to perform search in minimal time, with a few false positives and false negatives.

\section{REFERENCES}

[1] W.C. Priedhorsky, "Physical and economic limitations for distributed nuclear sensing," Journal of Nuclear Materials Management, 2006.

[2] A.Wald, "Sequential tests of statistical hypotheses," Annals of Mathematical Statistics, 16, 117-186, 1945.

[3] A. Klimenko, W.Priedhorsky, N.Hengartner, K.Borozdin, "Efficient Strategies for Low Statistics Nuclear Search," IEEE Trans. Nuc. Sci., 2006.

[4] A.Kumar, H.Tanner, A.Klimenko, K.Borozdin and W.Priedhorsky, "Automated Sequential search for Weak Radiation Sources," $14^{\text {th }}$ International Conference for Control and Automation, Ancona, Italy, 2006. 